\title{
A Charge-Sharing Bandpass Filter Topology with Boosted Q-Factor in 40-nm CMOS
}

\author{
Filipe D. Baumgratz ${ }^{*} \ddagger$, Sandro B. Ferreira ${ }^{\dagger}$, Michiel Steyaert ${ }^{\ddagger}$, Sergio Bampi ${ }^{*}$, and Filip Tavernier ${ }^{\ddagger}$ \\ * PGMicro, UFRGS, Porto Alegre, Brazil \\ Email: fdbaumgratz@inf.ufrgs.br \\ $\dagger$ PPGEE / itt Chip, Unisinos University, Sao Leopoldo, Brazil \\ $\ddagger$ ESAT-MICAS, KU Leuven, Leuven, Belgium
}

\begin{abstract}
This paper presents a detailed design of an innovative discrete-time charge-sharing bandpass filter with a feedback technique for boosting its quality factor. The design is implemented in 40-nm bulk CMOS, and it was integrated in silicon as part of a complete super-heterodyne receiver. In the proposed topology, cross-connected transconductors are used to boost the quality factor. The resulting selectivity of the complex filter is equivalent to a higher order filter but without the power consumption burden. The passive filter is fully programmable, which allows for an intermediate frequency selection in the range of $20 \mathrm{MHz}$ to $100 \mathrm{MHz}$. The consequent variable bandwidth enables its use for either narrowband or wideband applications. The passive filter, with transconductance amplification and clock generation, occupies an area of $0.24 \times 0.28 \mathrm{~mm}^{2}$ with a power consumption of $8 \mathrm{~mW}$ when operating with a $500 \mathrm{MHz}$ sampling frequency.
\end{abstract}

Index Terms - charge-sharing bandpass filter, discrete-time, noise, switched-capacitor.

\section{INTRODUCTION}

For many years, the basic choice of a receiver (RX) architecture has been direct conversion (DC) or a low-intermediatefrequency (IF) at most. The main reason for this preference has been usually the easier integration of the low-pass filters and the lack of image in the DC implementation, or the easier baseband filtering in the case of the low-IF implementation. Nevertheless, complex strategies were sometimes required to overcome the typical issues of $2^{\text {nd }}$ order non-linearity, flicker noise and DC offsets [1]-[3].

Recently, the super-heterodyne architecture has also become an option with the advent of high-Q bandpass filter (BPF) integration, avoiding the large external filters required to solve the image proplem in the past. The introduction of N-path filters in the RF front-end led the way to the start of this new revived interest in super-heterodyne receivers [4]. The N-path "complex" implementation can successively attenuate the RF image and, most important, it benefits directly from technology scaling, as it is implemented only with MOS switches and capacitors, all controlled by digital full-swing clocks. The main drawback presented by this solution are the filter replicas at the sampling rate as a consequence of the discrete-time (DT) nature of the filter, which are then folded back to the band of interest. This "replica" folding issue was solved by the fullrate charge-sharing (CS) $\mathrm{BPF}$ introduced in [5]. In [6], an enhancement to the full-rate CS-BPF was introduced, which in turn allows for a sharper filtering without additional power consumption burden.

In this paper, the modified CS-BPF which was tested in [6] has its CMOS design presented in detail. Its main advantages over the traditional CS-BPF approach are discussed, and the small noise impact of the circuit modification is carefully analysed. Section II reviews and generalizes the CS-BPF DT architecture. The modified CS-BPF and the noise analysis are presented in Section III. The filter architecture, simulation results and receiver measurements are presented in Sections IV and V, and the paper conclusions are drawn in Section VI.

\section{CHARGE-SHARING BANDPASS FILTER}

The basic charge-sharing bandpass filter (CS-BPF) is synthesized from the $4^{\text {th }}$-order Infinite Impulse Response (IIR) lowpass filter (LPF) [7]. To better understand this process, we start by the well-known first order IIR LPF with current input [8], shown in fig. 1(a). In this circuit, an input charge packet is stored in a history capacitor $\left(C_{H}\right)$, and on phase $\phi_{1}$, partially transferred to a previously discharged rotating capacitor $\left(C_{R}\right)$, acting as a lossy component. The order of the IIR LPF can be easily increased by adding more $C_{H}$ capacitors to share the charge stored in $C_{R}$ during subsequent phases, $\phi_{1}, \phi_{2}$ and so forth [8]. Hence, as shown in fig. 1(b), a $4^{\text {th }}$-order IIR LPF is created by sharing the charge of $C_{R}$ with three other $C_{H}$ [9].

The basic CS-BPF is created by connecting four inputs with increasing 90-degree phase shifts, to the $4^{\text {th }}$-order IIR LPF, as shown in fig. 1(c). Therefore, each $C_{H}$ stores the input charge of a different input, which is sampled by $C_{R}$ on one phase and shared with the next $C_{H}$ on the next phase [7]. In order to create a full-rate $1^{\text {st }}$-order CS-BPF, four basic cells of the CS-BPF are connected, as shown in fig. 1(d) [7], with the four $25 \%$ duty-cycle clocks phases in fig. 1(e). In [10], a generic CS-BPF has been presented and named as M/N-phase CS$\mathrm{BPF}$, where $\mathrm{M}$ is the number of inputs and $\mathrm{N}$ the number of phases. This nomenclature will be used hereafter.

Based on the schematic of the 4/4-phase CS-BPF presented in fig. 1(d), the transfer function is given by [7]

$$
H(z)=\frac{k}{\left(1-a z^{-1}\right)-e^{j \frac{\pi}{2}}(1-a) z^{-1}},
$$




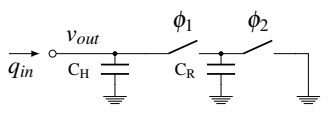

(a)

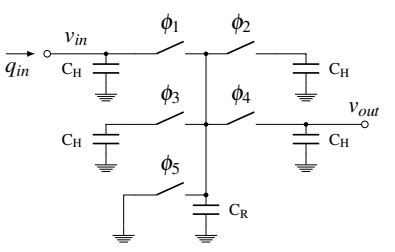

(b)

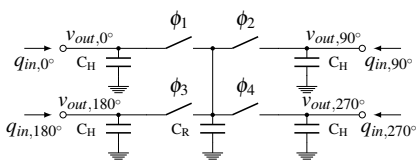

(c)

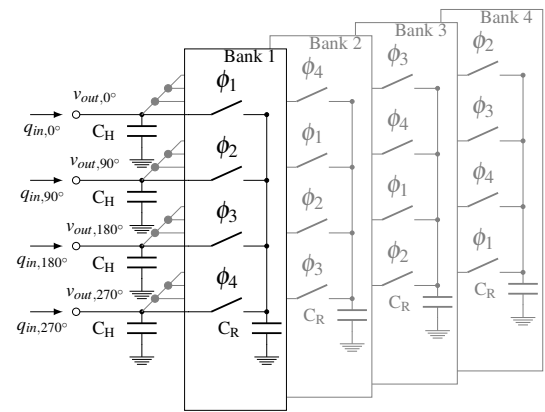

(d)

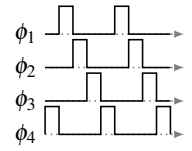

(e)
Fig. 1. (a) The $1^{\text {st }}$-order and (b) $4^{\text {th }}$ order IIR LPF. (c) The CS-BPF basic cell and (d) $1^{\text {st }}$-order full-rate CS-BPF. (e) The $25 \%$ clock, which drives the 4/4-phase CS-BPF filter.

where $k=1 /\left(C_{R}+C_{H}\right)$ and $a=C_{H} /\left(C_{H}+C_{R}\right)$. The filter Q-factor obtained from (1) is ideally 0.5 and can be easily improved in two different ways: by increasing the number of inputs (fig. 2(a)), or by adding lowpass poles (fig. 2(b)). To include these variations, a more general CS-BPF transfer function is given by (2).

$$
H(z)=\frac{k\left[(1-a) z^{-1}\right]^{N / M-1}}{\left(1-a z^{-1}\right)^{N / M}-e^{j \frac{2 \pi}{M}}\left[(1-a) z^{-1}\right]^{N / M}} .
$$

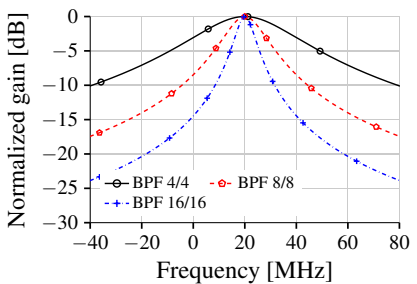

(a)

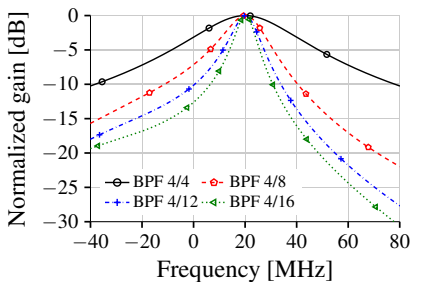

(b)
Fig. 2. (a) Filtering characteristic of 4/4-phase, 8/8-phase, and 16/16-phase CS-BPF;(b) Filtering characteristic of 4/4-phase, 4/8-phase, 4/12-phase, and 4/16-phase CS-BPF.

Based on (2), the transfer functions of two Q-factor enhancing alternatives are presented in fig. 2(a): 8/8-phase, 16/16phase, and in fig. 2(b): 4/8-phase, 4/12-phase, and 4/16-phase CS-BPF. In comparison to the 4/4-phase CS-BPF, the $8 / 8$ phase CS-BPF has a better filtering characteristic but the attenuation far from the central frequency is limited. This limitation is not presented by higher order filters like the $4 / 8$, $4 / 12$, and so forth, which can provide strong attenuation both close and far from the central frequency (fig. 2(b)). The basic cell implementations of the 8/8-phase and the 4/8-phase CSBPF alternatives are presented in fig. 3(a) [10] and fig. 3(b) [11]. To obtain the full-rate equivalents, $8 C_{R} \mathrm{~s}$ are placed in parallel, in a similar fashion to fig. 1(d).

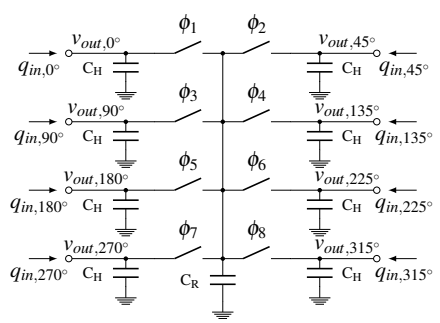

(a)

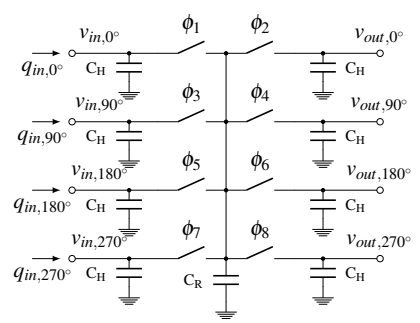

(b)
Fig. 3. (a) The schematic of a 8/8-phase CS-BPF and (b) a 4/8-phase CS-BPF.

Regardless of the number of inputs and the order, the central frequency of the CS-BPF is solely controlled by the ratio of the capacitors $C_{H}$ and $C_{R}$. This is the main advantage of the CS-BPF over the other bandpass filters since the capacitors in modern CMOS technologies are very robust to mismatch which plagues traditional topologies based on $\mathrm{Gm}-\mathrm{C}$, active$\mathrm{RC}$, or biquad. The CS-BPF central frequency is given by [10]

$$
f_{c}=\frac{f_{s}}{2 \pi} \arctan \left[\frac{(1-a) \sin (2 \pi / N)}{a+(1-a) \cos (2 \pi / N)}\right] .
$$

\section{MODIFIED CHARGE-SHARING BANDPASS FILTER}

Even though both methodologies are very effective to increase the Q-factor of the CS-BPF, they also increase the overall number of switches, the number of clock buffers, and consequently the power consumption. For instance, considering only the number of switches, the power consumption of the 4/8-phase BPF is $4 \mathrm{x}$ higher than the 4/4-phase BPF. Also, the switches of the 4/8-phase BPF work at a 2x-higher sampling rate. Hence, each of the clock generation circuits is going to consume $2 \mathrm{x}$ more power. Additionally, two other issues might arise from the increase of the filter order and the number of inputs. Firstly, in both cases, the sampling frequency of the CS-BPF increases. Hence, the switches must be proportionally faster, or the filter performance will decrease. Secondly, as the order of the CS-BPF increases, a secondary peak appears. This second peak is barely noticeable on the 4/8-phase CS-BPF transfer function (TF), but it already affects the symmetry of the magnitude TF. Due to the second peak, the image attenuation improves only by $5 \mathrm{~dB}$ from the 4/8phase to the 4/16-phase BPF [6]. Based on these points, we conclude that increasing the order of the filter beyond two (e.g., 4/8-phase BPF) is hardly worth the cost of complexity and power consumption.

Another possibility to enhance the Q-factor is by adding a pair of cross-connected transconductors at both the in-phase (I) and quadrature (Q) inputs of the filter. In fact, this crossconnected topology works as a negative impedance. Fig. 4 shows the schematic of a $4 / 8 \mathrm{CS}-\mathrm{BPF}$ modified using this 
negative impedance. By improving the Q-factor, both the image attenuation and the filtering of out-of-band blockers are improved. Nevertheless, since the I and Q paths are still independent, the I/Q mismatch remains affected mainly by the clock generation [6], [12].

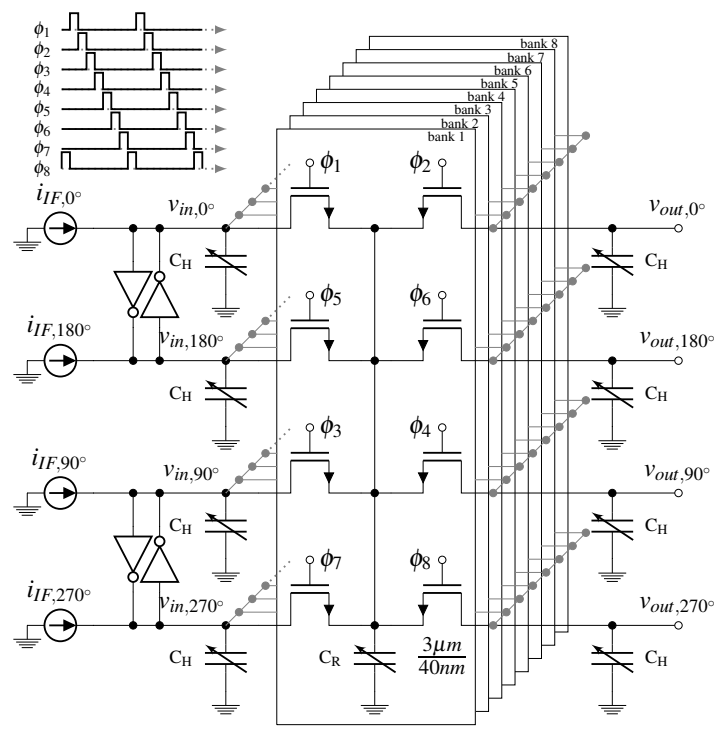

Fig. 4. Modified 4/8 phase CS BPF Schematics.

\section{A. Implementation of the modified 4/8-phase CS-BPF}

The negative impedance was implemented with inverters as presented in fig. 4. Based on this schematic, the time-domain input and output voltages of the modified 4/8-phase BPF at $t=n T_{S}$ are respectively

$$
\begin{aligned}
v_{\text {in }, i}[n] & =a v_{\text {in }, i}[n-1]+(1-a) v_{\text {out }, i-90^{\circ}}[n-1] \\
& +k q_{i}[n]+\beta(1-a) v_{\text {in }, i-180^{\circ}}[n], \\
v_{\text {out }, i}[n] & =a v_{\text {out }, i}[n-1]+(1-a) v_{\text {in }, i}[n-1],
\end{aligned}
$$

where $i \in\left\{0^{\circ}, 90^{\circ}, 180^{\circ}, 270^{\circ}\right\}$ and represents the phase of the input or output, and $\beta$ is the gain of the feedback loop used to implement the negative impedance. By converting (4) and (5) from the time-domain to the z-domain, the transfer function below is obtained.

$$
H(z)=\frac{k(1-a) z^{-1}}{\left(1-a z^{-1}\right)^{2}\left(1+\frac{1-a}{1-a z^{-1}} \beta\right)-j(1-a)^{2} z^{-2}}
$$

Fig. 5(a) shows the plotted transfer function of the modified 4/8-phase CS-BPF. By setting $\beta$ to -0.5 , according to the figure, an attenuation higher than $35 \mathrm{~dB}$ at the image frequency would be obtained, which is even better than the 4/16-phase CS-BPF case. However, $|\beta|<0.5$ should be adopted to ensure stability [6], and the CS-BPF would operate close to the oscillation condition. Fig. 5(b) presents a zoomed version of the pole representation of (6) as $\beta$ is varied, showing that one of the poles is crossing the unit circle at $\beta=-0.5$.

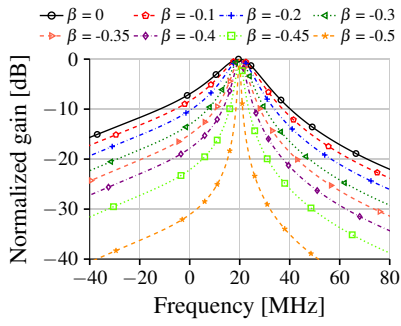

(a)

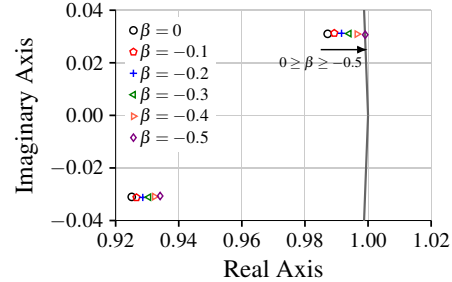

(b)
Fig. 5. (a) Modified 4/8-phase CS BPF transfer function;(b) Pole-zero mapping of the modified 4/8-phase CS BPF TF (6).

\section{B. Noise analysis of the modified charge-sharing bandpass filter}

In addition to stability, noise is a concern when the negative impedance is added to the filter. Based on the noise analysis of the 4/4-phase CS-BPF presented in [7], the noise contribution of the modified 4/8-phase CS-BPF is presented hereafter.

The noise sources of the filter are the switches and the inverters of the negative impedance, with thermal noise power spectral density (PSD) given, respectively, by (7) and (8).

$$
\begin{gathered}
S_{s w}(f)=4 k T R_{O N} \\
S_{i n v}(f)=4 k T \gamma / G_{m},
\end{gathered}
$$

where $\mathrm{k}$ is the Boltzmann constant, $\mathrm{T}$ is the absolute temperature, $R_{O N}$ is the resistance when the switch is closed, $\gamma$ is the noise parameter, and $G_{m}$ is the overall transconductance of the inverter. However, due to the sampling process, the noise from frequencies above $f_{s} / 2$ is folded to the interval 0 -to$f_{s} / 2$. Hence, after taking the folding into consideration, the sampled noise PSD of the switch and the inverter are given by (9) and (10), respectively [13].

$$
\begin{gathered}
S_{s w, S / H}(f)=\frac{2 k T}{C_{R} f_{s}}, \quad 0 \leq f \leq f_{s} / 2 \\
S_{i n v, S / H}(f)=\frac{2 k T \gamma}{G_{m} C_{R} f_{s} R_{O N}} . \quad 0 \leq f \leq f_{s} / 2
\end{gathered}
$$

The sampled noise PSDs will be considered as the noise sources in the following analysis.

Fig. 6 shows the simplified noise model, which considers no input charge packets. The purpose of this analysis is to compare the noise of the 4/8-phase CS-BPF with and without the addition of the negative impedance. First, the noise contribution of each noise source to each output is calculated. In fact, since the circuit is symmetrical, the noise at all outputs are equal, and we need to only compute for one output.

The noise sources are uncorrelated, so superposition can be used to compute the contribution of each source to the output noise. Therefore, to calculate the output noise due to $v_{n 1}$, the other noise sources are set to zero. The time-domain noise of the first output and input at $t=n T_{s}$ is given by

$$
v_{\text {out }, 0^{\circ}}[n]=a v_{\text {out }, 0^{\circ}}[n-1]+b v_{\text {in }, 0^{\circ}}[n-1]-b v_{n 1}[n-1] \text {, }
$$




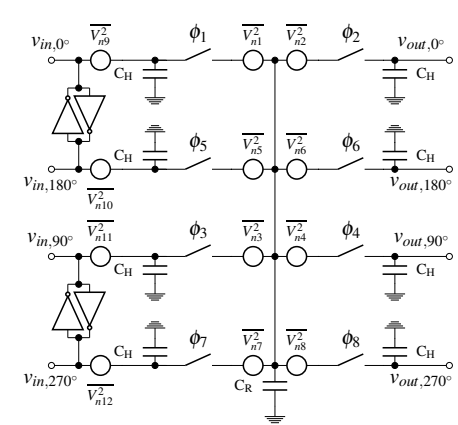

Fig. 6. Noise sources of the modified 4/8-phase CS-BPF.

$$
\begin{aligned}
v_{i n, 0^{\circ}}[n]=a v_{i n, 0^{\circ}}[n-1] & +b v_{o u t, 270^{\circ}}[n-1] \\
& +\beta b v_{i n, 180^{\circ}}[n]+b v_{n 1}[n],
\end{aligned}
$$

where $b=1-a$, and $v_{n 1}=\sqrt{\overline{V_{n 1}^{2}}}$. The time-domain noise equations of the other three inputs and outputs are

$$
\begin{array}{r}
v_{\text {out }, i}[n]=a v_{\text {out }, i}[n-1]+b v_{\text {in }, i}[n-1], \\
v_{i n, i}[n]=a v_{\text {in }, i}[n-1]+ \\
b v_{o u t, i-90^{\circ}}[n-1] \\
+\beta b v_{i n, i-180^{\circ}}[n],
\end{array}
$$

where $i \in\left\{90^{\circ}, 180^{\circ}, 270^{\circ}\right\}$. By converting (11) - (14) from the time-domain to the z-domain, the noise transfer function of $v_{n 1}$ to each output is

$$
\begin{aligned}
& H_{1}=\frac{\left(1-a z^{-1}\right)^{7} b z^{-1}-\left(1-a z^{-1}\right)^{6} b^{2} z^{-1}+A_{1}}{-\left(1-a z^{-1}\right)^{8}+b^{8} z^{-8}+B_{1}}, \\
& H_{2}=\frac{\left(1-a z^{-1}\right)^{5} b^{3} z^{-3}-\left(1-a z^{-1}\right)^{4} b^{4} z^{-3}+A_{2}}{-\left(1-a z^{-1}\right)^{8}+b^{8} z^{-8}+B_{1}} \\
& H_{3}=\frac{\left(1-a z^{-1}\right)^{3} b^{5} z^{-5}-\left(1-a z^{-1}\right)^{2} b^{6} z^{-5}+A_{3}}{-\left(1-a z^{-1}\right)^{8}+b^{8} z^{-8}+B_{1}} \\
& H_{4}=\frac{\left(1-a z^{-1}\right) b^{7} z^{-7}-b^{8} z^{-7}+A_{4}}{-\left(1-a z^{-1}\right)^{8}+b^{8} z^{-8}+B_{1}}
\end{aligned}
$$

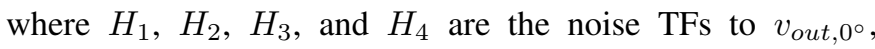
$v_{\text {out }, 90^{\circ}}, v_{\text {out }, 180^{\circ}}$, and $v_{\text {out }, 270^{\circ}}$, respectively. Also, the terms $A_{1}, A_{2}, A_{3}, A_{4}$, and $B_{1}$ are introduced by the negative impedance and presented below:

$$
\begin{aligned}
A_{1} & =\left[-2\left(1-a z^{-1}\right)+b\right]\left[\left(1-a z^{-1}\right)^{4} b^{3} \beta^{2} z^{-1}\right. \\
& \left.+\left(1-a z^{-1}\right) b^{6} \beta z^{-5}\right]+\left(1-a z^{-1}\right)^{3} b^{5} \beta^{4} z^{-1}, \\
A_{2} & =-\left(1-a z^{-1}\right)^{3} b^{5} \beta^{2} z^{-3} \\
& -\left(1-a z^{-1}\right)^{2} b^{6} \beta^{2} z^{-3}-b^{8} \beta z^{-7}, \\
A_{3} & =-\left(1-a z^{-1}\right)^{5} b^{3} \beta z^{-1}+\left(1-a z^{-1}\right)^{3} b^{5} \beta^{3} z^{-1} \\
& +\left(1-a z^{-1}\right) b^{7} \beta^{2} z^{-5} \\
A_{4} & =-\left[-\left(1-a z^{-1}\right)^{2}-2\left(1-a z^{-1}\right) b-\beta^{2} b^{2}\right] \\
& \left(1-a z^{-1}\right)^{2} \beta b^{4} z^{-3} \\
B_{1} & =2\left(1-a z^{-1}\right)^{6} b^{2} \beta^{2}-\left(1-a z^{-1}\right)^{4} b^{4} \beta^{4}
\end{aligned}
$$


of the switches and inverters. Since the negative impedance enhances the gain of the filter, a slight reduction of the noise is observed, and a small impact in the overall RX noise figure is expected.

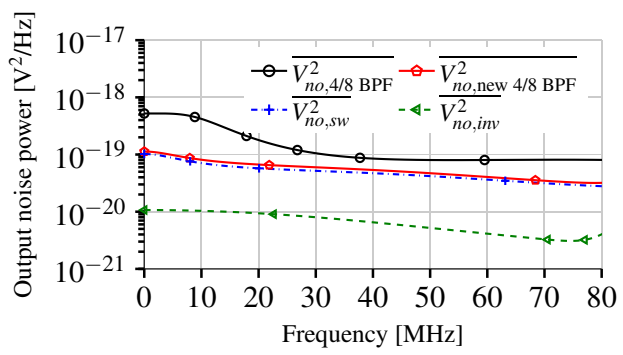

Fig. 7. The output noise PSD calculation of the 4/8-phase BPF with and without negative impedance are presented with solid lines. Dashed and dasheddotted lines show the output noise of the switches and inverters of the modified 4/8-phase BPF.

\section{Circuit implementation}

The modified 4/8-phase CS-BPF was implemented and measured as the second filtering stage of a complete receiver presented in fig. 8 [6].

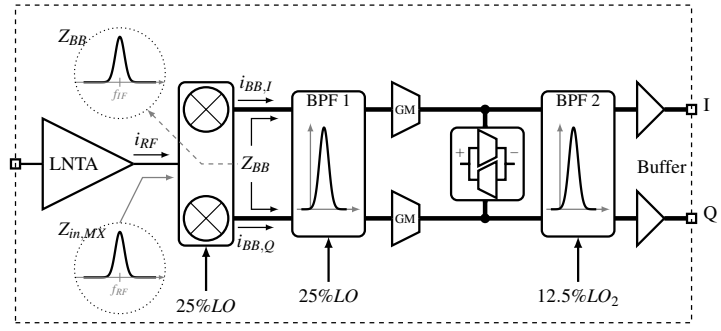

Fig. 8. Simplified block diagram of the complete implemented receiver.

The receiver in fig. 8 is composed of two basic stages: a $\mathrm{RF}$ front-end and a intermediate frequency (IF) filtering stage.

The RF front-end is composed of wideband LNTA, 25\% duty-cycle sampling mixer, 4/4 full-rate CS-BPF which operates at the mixer sampling rate, and clock generation circuits. The front-end is a super-heterodyne architecture covering the frequency range from $500 \mathrm{MHz}$ to $4 \mathrm{GHz}$, with variable intermediate frequency (IF) and adjustable channel pass-band. It is mainly in charge of low-noise amplification and out-ofband filtering, and was presented in detail in [6].

The second stage is composed of a basic "inverter-like" transconductor (Gm-cell) stage detailed in fig. 9 and a modified 4/8-phase CS-BPF (fig. 4) operating at a fixed 500-MHz sampling rate. The $\mathrm{Gm}$-cell gives a gain of $12.8 \mathrm{~dB}$ when combined with the filter input resistance.

The eight $12.5 \%$ duty-cycle clock phases required by the filter are generated by the frequency divider-by-4 (fig. 10(a)). The latches presented in fig. 10(b) are implemented using tristate inverters (fig. 10(c)) in order to achieve sharp transitions.

\section{RESUlts}

The DT filter was implemented in silicon as part of the receiver designed and fabricated in TSMC 40-nm CMOS

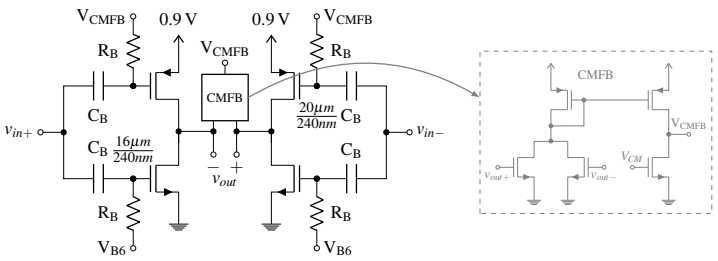

Fig. 9. "Inverter-like" transconductor.

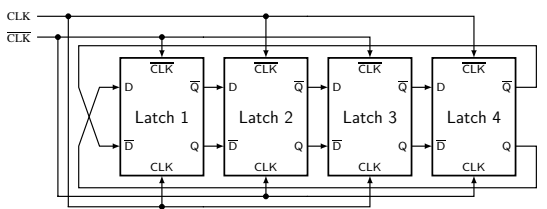

(a)

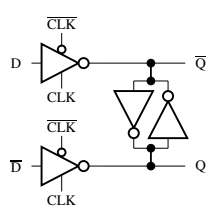

(b)

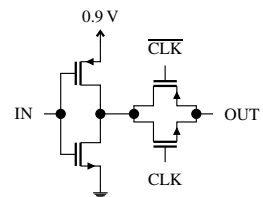

(c)
Fig. 10. (a) The divider-by-4 that generates the $12.5 \%$ non-overlaping clock, (b) the tristate latchs, and (c) the tristate circuit.

(fig. 11) [6]. The filter, the Gm-cell and the 8-phase clock generation circuit jointly occupy a slicon area of $0.24 \times 0.28$ $\mathrm{mm}^{2}$.

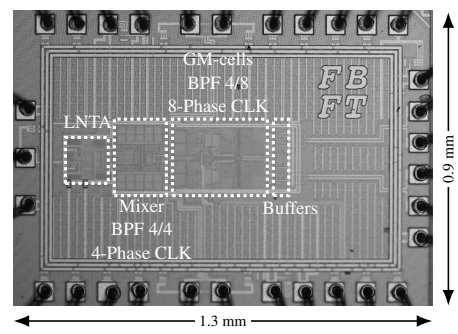

Fig. 11. Chip micrograph

The filter implementation is detailed in fig. 4. The feedback gain $\beta$ is implemented as $G_{m} \times R_{i n}$, where $G_{m}$ is the inverter transcondutance, and $R_{i n}$ is the input resistance of the filter, $R_{i n} \approx 1 / f_{s} C_{R} . C_{H}$ and $C_{R}$ are implemented using 8-bit binary-programmed capacitor banks, ranging from $600 \mathrm{fF}$ to $9 \mathrm{pF}$ and $25 \mathrm{fF}$ to $375 \mathrm{fF}$, respectively. According to (3), the resolution of the capacitor banks allow for center frequency programming in the range of $20 \mathrm{MHz}$ to 60 $\mathrm{MHz}$, which creates a variable passband characteristic due to the constant quality-factor presented by our filter design. Consequently, the filter is suitable for both wideband and narrowband applications. For instance, the $20 \mathrm{MHz}$ bandwidth required by $791-821 \mathrm{MHz}$ and $3600-3800 \mathrm{MHz}$ LTE bands can be easily achieved by moving the IF to $-40 \mathrm{MHz}$ as shown in the extracted simulations (fig. 12).

Fig. 13(a) shows the improvement in selectivity of the $\beta=-0.2$ modified filter vs the traditional $4 / 8$-phase CS-BPF approach. The feedback is used here to compensate for losses 


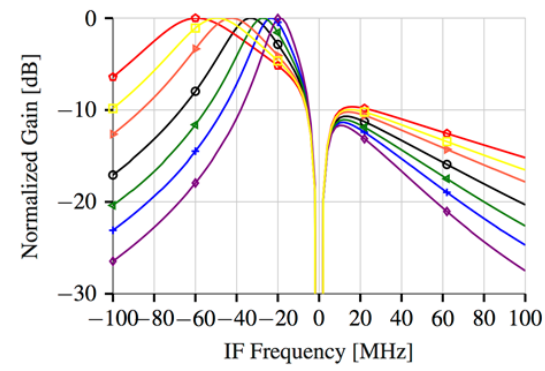

Fig. 12. Extracted simulation of the Gm and the modified 4/8-phase CS-BPF center frequency programming.

in the CS-BPF implementation, which brings the filter transfer function closer to the ideal implementation. As expected by the noise analysis, noise figure is not strongly impacted by the modification (fig. 13(b)).

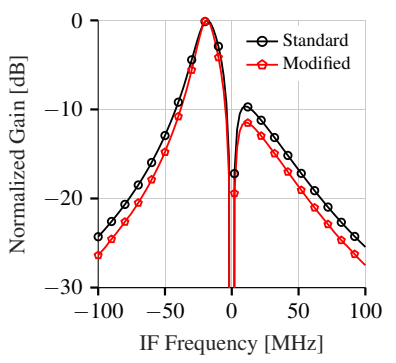

(a)

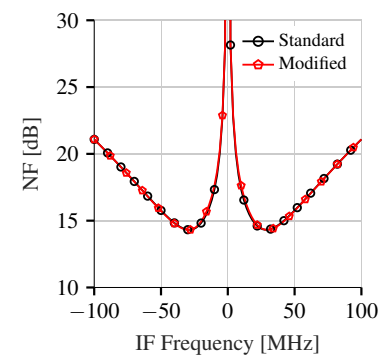

(b)
Fig. 13. (a) Normalized gain and (b) Noise figure of the standard and modified 4/8-phase CS-BPF with $|\beta|=0.2$.

Fig. 14(a) and fig. 14(b) present measured gain and noise, respectively, for two chip samples of the complete receiver. Good selectivity as well as good matching characteristics are shown, resulting from the modified CS-BPF, as can be easily observed in the figure with an attenuation of about $16 \mathrm{~dB}$ at the image frequency. The measured power consumption of the Gm-cell with $12.8 \mathrm{~dB}$ of gain and the clock generation circuits operating at $500 \mathrm{MHz}$ are $2.7 \mathrm{~mW}$ and $5.35 \mathrm{~mW}$ respectively, from a $0.9 \mathrm{~V}$ power supply.

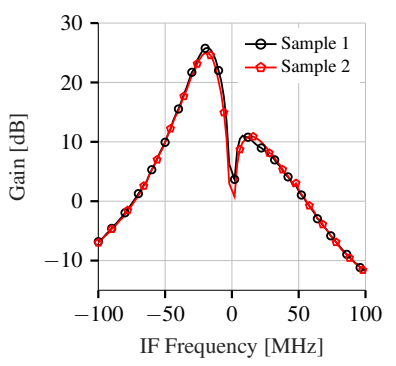

(a)

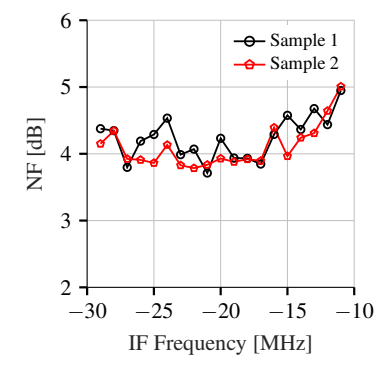

(b)
Fig. 14. (a) Gain and (b) Noise Figure measurement results with $1 \mathrm{GHz}$ LO frequency for the complete implemented RX.

\section{CONCLUSION}

This paper presented a detailed analysis of a newly modified charge-sharing bandpass filter. The discrete-time filter is fully-programmable, which allows for both intermediate frequency and passband adjustments. This flexibility makes this technique a good candidate for narrowband and wideband RF transceiver applications. The charge-sharing bandpass filter topology is modified using cross-connected transconductors at the filter inputs which enables higher selectivity without increasing complexity, noise and power consumption. The filter is implemented in 40-nm bulk CMOS and it occupies an area of $0.24 \times 0.28 \mathrm{~mm}^{2}$, considering the transconductor amplifier, and the clock generation circuit area altogether. The three blocks consume $8 \mathrm{~mW}$ from a $0.9 \mathrm{~V}$ DC power supply.

\section{ACKNOWLEDGMENT}

The authors would like to thank the CNPq, CAPES, and FAPERGS Agencies for partially funding this research.

\section{REFERENCES}

[1] J. Borremans, G. Mandal, V. Giannini, B. Debaillie, M. Ingels, T. Sano, B. Verbruggen, and J. Craninckx, "A $40 \mathrm{~nm}$ CMOS 0.4-6 GHz Receiver Resilient to Out-of-Band Blockers," IEEE Journal of Solid-State Circuits, vol. 46, no. 7, pp. 1659-1671, July 2011.

[2] B. van Liempd, J. Borremans, E. Martens, S. Cha, H. Suys, B. Verbruggen, and J. Craninckx, "A 0.9 V 0.4-6 GHz Harmonic Recombination SDR Receiver in $28 \mathrm{~nm}$ CMOS With HR3/HR5 and IIP2 Calibration," IEEE Journal of Solid-State Circuits, vol. 49, no. 8, pp. 1815-1826, Aug 2014.

[3] D. Murphy, H. Darabi, and H. Xu, "A noise-cancelling receiver resilient to large harmonic blockers," IEEE Journal of Solid-State Circuits, vol. 50, no. 6, pp. 1336-1350, June 2015.

[4] A. Mirzaei, H. Darabi, and D. Murphy, "A Low-Power Process-Scalable Super-Heterodyne Receiver With Integrated High- $Q$ Filters," IEEE Journal of Solid-State Circuits, vol. 46, no. 12, pp. 2920-2932, Dec 2011.

[5] M. Tohidian, I. Madadi, and R. B. Staszewski, "A fully integrated highly reconfigurable discrete-time superheterodyne receiver," in 2014 IEEE International Solid-State Circuits Conference Digest of Technical Papers (ISSCC), Feb 2014, pp. 1-3.

[6] F. D. Baumgratz, S. B. Ferreira, M. S. J. Steyaert, S. Bampi, and F. Tavernier, "40-nm CMOS Wideband High-IF Receiver Using a Modified Charge-Sharing Bandpass Filter to Boost Q-Factor," IEEE Transactions on Circuits and Systems I: Regular Papers, pp. 1-11, 2018.

[7] I. Madadi, M. Tohidian, and R. B. Staszewski, "Analysis and Design of I/Q Charge-Sharing Band-Pass-Filter for Superheterodyne Receivers," IEEE Transactions on Circuits and Systems I: Regular Papers, vol. 62, no. 8, pp. 2114-2121, Aug 2015.

[8] G. Hueber and R. B. Staszewski, Multi-mode/multi-band RF transceivers for wireless communications: advanced techniques, architectures, and trends. New Jersey,USA: John Wiley \& Sons, Inc., 2011.

[9] M. Tohidian, I. Madadi, and R. B. Staszewski, "Analysis and Design of a High-Order Discrete-Time Passive IIR Low-Pass Filter," IEEE Journal of Solid-State Circuits, vol. 49, no. 11, pp. 2575-2587, Nov 2014.

[10] I. Madadi, M. Tohidian, K. Cornelissens, P. Vandenameele, and R. B. Staszewski, "A High IIP2 SAW-Less Superheterodyne Receiver With Multistage Harmonic Rejection," IEEE Journal of Solid-State Circuits, vol. 51, no. 2, pp. 332-347, Feb 2016.

[11] F. W. Kuo, S. B. Ferreira, H. N. R. Chen, L. C. Cho, C. P. Jou, F. L. Hsueh, I. Madadi, M. Tohidian, M. Shahmohammadi, M. Babaie, and R. B. Staszewski, "A Bluetooth Low-Energy Transceiver With 3.7-mW All-Digital Transmitter, 2.75-mW High-IF Discrete-Time Receiver, and TX/RX Switchable On-Chip Matching Network," IEEE Journal of SolidState Circuits, vol. 52, no. 4, pp. 1144-1162, April 2017.

[12] S. B. Ferreira, F. W. Kuo, M. Babaie, S. Bampi, and R. B. Staszewski, "System Design of a 2.75-mW Discrete-Time Superheterodyne Receiver for Bluetooth Low Energy," IEEE Transactions on Microwave Theory and Techniques, vol. 65, no. 5, pp. 1904-1913, May 2017.

[13] R. Gregorian and G. C. Temes, Analog MOS Integrated Circuits for Signal Processing, 1st ed. New York: Wiley-Interscience, 1986. 